\title{
The autonomous frontal obstacle avoidance system with trajectory updating function
}

\author{
Ryuzo HAYASHI*, Shimpei FUJIMORI*, Hideki IWATA* and Masao NAGAI** \\ ${ }^{*}$ Department of Mechanical Engineering, Tokyo University of Science \\ 6-3-1 Niijuku, Katsushika-ku, Tokyo 125-8585, Japan \\ E-mail: hayashi@rs.tus.ac.jp \\ **Japan Automobile Research Institute \\ Jidosha Kaikan, 1-1-30 Shiba Daimon, Minato-ku, Tokyo, 105-0012, Japan
}

Received: 11 May 2016; Revised: 26 October 2016; Accepted: 19 January 2017

\begin{abstract}
Recently, in order to decrease the number of traffic accident, various automotive safety technologies have been studied. As one of those technologies, the authors have proposed the autonomous frontal obstacle avoidance system, which recognizes a frontal obstacle such as a pedestrian, predicts the location of collision based on the obstacle's motion and avoids collision by autonomous evasive steering. The proposed system calculates avoidance trajectory once at the start of avoidance. Therefore, it cannot handle the situation that the obstacle's motion changes. Especially in the case that the obstacle accelerates after its appearance, the system will cause a collision with the obstacle. Since pedestrians' motion in the real world is not always constant, change of motion of the obstacle must be considered. Therefore, in this paper, the authors improve the system so that it can handle those situations by adding an updating function of avoidance trajectory. This paper first describe the derivation of avoidance trajectory from the vehicle state in evasive motion. Next, the autonomous frontal obstacle system is redesigned by building peripheral functions such as collision forecasting functions and appropriate trajectory selection function and integrating them. The redesigned system is examined by numerical simulations and compared with the previously proposed systems to verify the validity and effectiveness. The results indicate that the system proposed in this paper is valid and more effective than the previously proposed system.
\end{abstract}

Key words : Automobile, Vehicle dynamics, Intelligent equipment, Motion control, Collision avoidance

\section{Introduction}

Popularization of active safety technologies for automobile such as ABS and ESC has contributed to a remarkable decrease of the number of traffic accidents; however, over 4,000 traffic fatalities a year still occur in Japan. To realize society of zero traffic accidents, further technological innovations are needed.

Since approximately $40 \%$ of drivers do not take any avoidance maneuver at the time of a traffic accident (ITARDA, 2005), automatic emergency braking systems (AEB) are now expected to reduce the number of accidents. In addition, since it has been reported that collision avoidance by steering is more effective at high speed (Horiuchi et al., 2006), collision avoidance technologies by evasive steering will also be effective to reducing the number of accidents.

Collision avoidance technologies by steering are still in the research phase. Many researches are being promoted from various viewpoint. For example, Glacer et al. (2010) have proposed a maneuver-based trajectory planning in which the avoidance trajectory is expressed as a polynomial. Soudbakhsh et al. (2014) also utilize a polynomial trajectory and proposed a trajectory optimization method to meet the limitation of lateral acceleration. Other researches include evasive motion control methods of the vehicle in which avoidance trajectories are not derived explicitly. Fujioka et al. (2008) proposed the optimal vehicle motion control method which minimizes the collision risk value and Ohmuro et al. (2010) proposed that which minimizes the resultant vehicle force. Shibata et al. (2014) utilize potential field method, which is commonly used in robotics. Fuzzy control has been also utilized in collision avoidance by steering (Llorca et al., 2011).

The authors have also proposed a collision avoidance technology using steering. The main feature is that it utilizes 
geometrically-derived arc trajectories as avoidance trajectories. It enables to calculate avoidance trajectories with very low computational cost, and forecast availability of collision avoidance in advance of starting autonomous collision avoidance maneuver. The autonomous frontal obstacle avoidance system was designed as a total collision avoidance system in which sensing of an obstacle, judgment of availability of avoidance, decision of an optimal avoidance method (braking or steering) and control of the vehicle motion are integrated (Hayashi et al., 2011). In addition, incorporation of prediction of obstacle's movement has improved the system to avoid collision with moving obstacles like pedestrians (Hayashi et al., 2012). However, the system needs more improvement from the following viewpoints. However, there are still remaining challenges causing decrement of collision avoidance performance. One is that trajectory tracking performance is not good because of the dynamics of the vehicle and its steering system. Another challenge is that it cannot respond to change of behavior of the obstacle during avoidance. These challenges have its roots in that the avoidance trajectory is generated only once at the start of the avoidance maneuver. Therefore, incorporating trajectory updating function in the system is considered to solve the challenges. The other remaining challenges include improvement of accuracy of pedestrian detection and motion prediction of it. However, since the system employs simple and basic methods for them, the system can be improved by replacing them with advanced techniques actively researched and developed by many researchers in recent years.

Based on the aforementioned, this paper proposes a regeneration method of avoidance trajectory which start from the vehicle position in the middle of avoidance. Then the autonomous frontal obstacle avoidance system is redesigned to incorporate trajectory updating function utilizing the proposed trajectory regeneration method. The redesigned system is examined by numerical simulations to evaluate the validity and effectiveness.

\section{Target scene and supposed vehicle}

The target situation of this study is shown in Fig. 1. A frontal obstacle such as a pedestrian jumps out from the roadside while the ego-vehicle is driving along road boundaries such as walls. Assumed conditions are as follows:

(1) The road is straight with road boundaries on both sides in an urban area.

(2) Only a single obstacle appears from the roadside when the ego-vehicle is in motion.

(3) It is not available to avoid the obstacle by evading leftward.

(4) There is a space between the obstacle and the right-side road boundary for the vehicle to pass through.

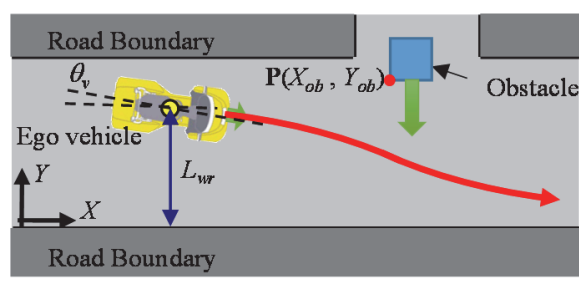

Fig. 1 Target Scene.

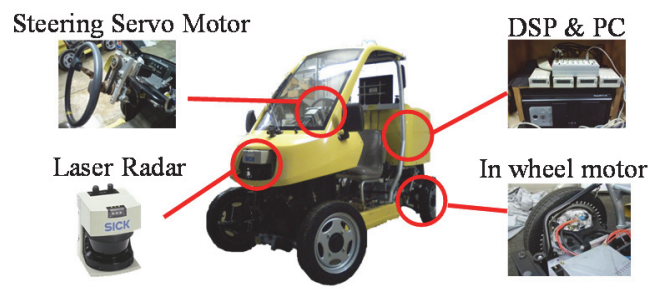

Fig. 2 Supposed vehicle.

Table 1 Specifications of the supposed vehicle.

\begin{tabular}{|l|c|r|l|}
\hline \multicolumn{1}{|c|}{ DEFINITION } & \multicolumn{1}{c|}{ SYMBOL } & \multicolumn{1}{c|}{ VALUE } & \multicolumn{1}{c|}{ UNIT } \\
\hline Weight & $m$ & 470 & $\mathrm{~kg}$ \\
\hline Length & $l_{v}$ & 2.365 & $\mathrm{~m}$ \\
\hline Width & $w$ & 0.995 & $\mathrm{~m}$ \\
\hline Wheelbase & $l$ & 1.71 & $\mathrm{~m}$ \\
\hline Length from front axle to center of gravity & $l_{f}$ & 0.90 & $\mathrm{~m}$ \\
\hline Length from rear axle to center of gravity & $l_{r}$ & 0.81 & $\mathrm{~m}$ \\
\hline Minimum turning radius & $R_{\min }$ & 3.3 & $\mathrm{~m}$ \\
\hline Yaw moment of inertia & $I_{z}$ & 311 & $\mathrm{kgm}^{2}$ \\
\hline Steering gear ratio & $N$ & 18.7 & \\
\hline Stability factor & $K$ & $6.31 \times 10^{-4}$ & \\
\hline
\end{tabular}




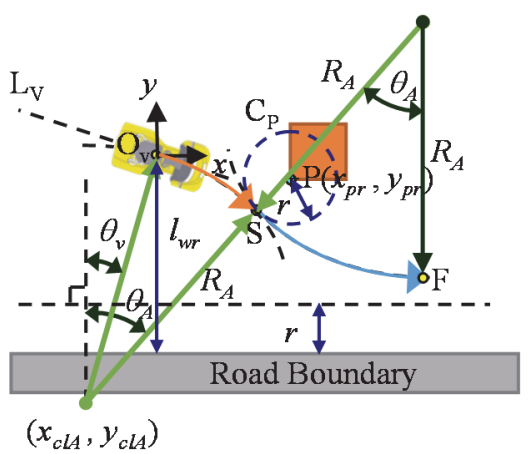

(a) Trajectory A

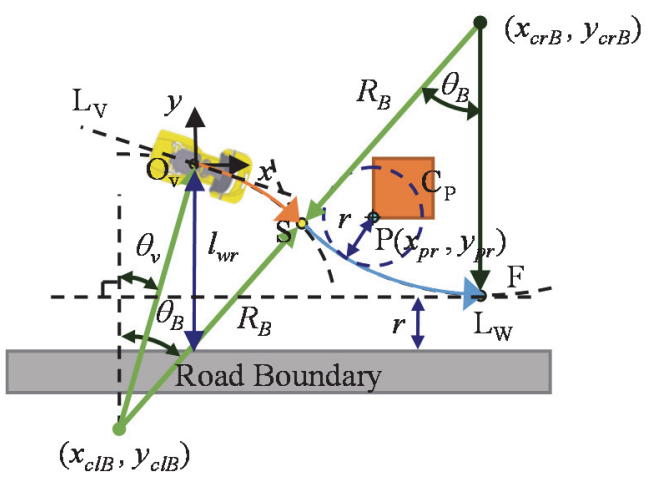

(b) Trajectory B

Fig. 3 Geometric description of the avoidance trajectory by two arcs.

The supposed vehicle is shown in Fig. 2. It is equipped with a laser range finder (LIDAR) to obtain the relative distances to objects over a wide range. It is also equipped with vehicle motion sensors such as accelerometers, a vehicle speed sensor and a yaw rate sensor. In addition, a digital signal processor is installed on the vehicle to control the vehicle velocity and steering angle. The specifications of the vehicle is shown in Table 1.

\section{The theory of avoidance trajectory}

\subsection{The number of arcs needed to evade an obstacle and a road boundary}

This chapter describes the derivation of avoidance trajectories, which is the essential of this study. The derivation of avoidance trajectory differs depending on positional relationship among the vehicle, the obstacle and the road boundary. The positional relationship is divided roughly into two types and they are subdivided into two patterns each.

\subsection{Avoidance trajectory by two arcs}

If the obstacle is on the vehicle's course, the vehicle should turn right to evade the obstacle first, and then turn left to evade the road boundary. Therefore, two turns are necessary to evade both the obstacle and the road boundary at least. In this study, to minimize the maximum steering angle during avoidance, the avoidance trajectory is made by two arcs with the same radius and the radius should be as large as possible.

The avoidance trajectory by two arcs can be subdivided into two patterns, as shown in Fig. 3. In Fig. 3, $\mathrm{O}_{\mathrm{v}}$ is current vehicle position, $\mathrm{S}$ is the steering switching point at which the vehicle should change turning direction from right to left, $\mathrm{F}$ is finishing position of avoidance. $\mathrm{F}$ is defined as the point at which the vehicle direction is in parallel to the road boundary.

Trajectory $\mathrm{A}$ is for cases that the road boundary is far enough from the vehicle and the obstacle (Fig. 3(a)). In this case, the vehicle should concentrate only on avoiding the obstacle at first. After avoiding the obstacle, it is desirable to restore the yaw angle of the vehicle. The geometrical condition of trajectory A is as follows:

(A-i) The arc $\mathrm{O}_{\mathrm{V}} \mathrm{S}$ is circumscribed to the vehicle direction line $\mathrm{L}_{\mathrm{V}}$,

(A-ii) The arc $\mathrm{O}_{\mathrm{V}} \mathrm{S}$ passes through the current vehicle position,

(A-iii) The off-limit circle $\mathrm{C}_{\mathrm{P}}$ is circumscribed to the arc $\mathrm{O}_{\mathrm{V}} \mathrm{S}$. (The off-limit circle $\mathrm{C}_{\mathrm{P}}$ is a circle drawn centered on the right edge of the obstacle with radius $r . r$ is the half width of the vehicle.)

From These conditions, turning radius $R_{A}$ and the central angle of the arc $\theta_{A}$ are derived as follows:

$$
R_{A}=\frac{x_{p r}{ }^{2}+y_{p r}{ }^{2}-r^{2}}{2\left(r-x_{p r} \sin \theta_{v}-y_{p r} \cos \theta_{v}\right)}, \quad \theta_{A}=\cos ^{-1}\left(\frac{R_{A} \cos \theta_{v}+y_{p r}}{R_{A}+r}\right),
$$

where $x_{p r}$ and $y_{p r}$ are coordinates of the right edge of the obstacle, $\theta_{v}$ is the angle of the vehicle against the road boundary. The arc SF is as the arc with radius $R_{A}$ which starts from the point $\mathrm{S}$ and ends in parallel to the road boundary. Trajectory $\mathrm{A}$ is available when the finishing point $\mathrm{F}$ is more than $r$ away from the road boundary. This is expressed as: 


$$
R_{A}\left(1-2 \cos \theta_{A}+\cos \theta_{v}\right) \leq l_{w r}-r
$$

where $l_{w r}$ is the distance between the vehicle and the road boundary.

If Eq. (2) is not satisfied, avoidance by trajectory A causes a collision with the road boundary. In such cases, trajectory B (Fig. 3(b)) is able to avoid the obstacle without having a collision with the road boundary. Trajectory B is formulated with the following geometrical conditions.

(B-i) The arc $\mathrm{O}_{V} \mathrm{~S}$ is circumscribed to the vehicle direction line $\mathrm{L}_{V}$.

(B-ii) The arc $\mathrm{O}_{V} \mathrm{~S}$ passes through the current vehicle position.

(B-iii) Radius of the arc $\mathrm{O}_{\mathrm{V}} \mathrm{S}$ equals to that of the arc SF.

(B-iv) The arc $\mathrm{O}_{\mathrm{V}} \mathrm{S}$ is circumscribed to the arc SF at the steering switching point $\mathrm{S}$.

(B-v) The arc SF is circumscribed to the off-limit line $\mathrm{L}_{\mathrm{W}}$.

(B-vi) The off-limit circle CP is inscribed in the arc SF.

From these conditions, the following fourth degree equation is obtained:

$$
A R_{B}^{4}+B R_{B}^{3}+C R_{B}^{2}+D R_{B}+E=0
$$

where

$$
\left.\begin{array}{l}
A=a^{2}-4\left\{4-\left(1+\cos \theta_{v}\right)^{2}\right\} \sin ^{2} \theta_{v}, \\
B=2 a b-8\left\{4-\left(1+\cos \theta_{v}\right)^{2}\right\} x_{p r} \sin \theta_{v}+8\left(1+\cos \theta_{v}\right)\left(-l_{w r}+r\right) \sin ^{2} \theta_{v}, \\
C=b^{2}+2 c a-4\left\{4-\left(1+\cos \theta_{v}\right)^{2}\right\} x_{p r}{ }^{2}+16\left(1+\cos \theta_{v}\right)\left(-l_{w r}+r\right) x_{p r} \sin \theta_{v}+4\left(-l_{w r}+r\right)^{2} \sin ^{2} \theta_{v}, \\
D=2 b c+8\left(1+\cos \theta_{v}\right)\left(-l_{w r}+r\right) x_{p r}{ }^{2}+8\left(-l_{w r}+r\right)^{2} x_{p r} \sin \theta_{v}, \\
E=4\left(-l_{w r}+r\right)^{2} x_{p r}{ }^{2}+c^{2},
\end{array}\right\}
$$

where

$$
\left.\begin{array}{l}
a=4\left(1+\sin ^{2} \theta_{v}\right)-\left(1+\cos \theta_{v}\right)^{2} \\
b=2 x_{p r} \sin \theta_{v}-2\left(1+\cos \theta_{v}\right)\left(-l_{w r}+r\right)+2\left(y_{p r}-l_{w r}+r\right)+2 r \\
c=x_{p r}{ }^{2}-\left(-l_{w r}+r\right)^{2}+\left(y_{p r}+l_{w r}-r\right)^{2} .
\end{array}\right\}
$$

The radius $R_{B}$ is the largest real root among four roots of Eq. (4). It can be solved numerically with negligible computational cost. $\theta_{B}$ can be calculated by assigning the value of $R_{B}$ to the following equation:

$$
\theta_{B}=\cos ^{-1}\left(\frac{R_{B}\left(1+\cos \theta_{v}\right)+r-l_{w r}}{2 R_{B}}\right) .
$$

Trajectory $B$ is available unless the off-limit circle $C_{P}$ intersects the off-limit line $\mathrm{L}_{\mathrm{W}}$, that is, there is no space to pass between the obstacle and the road boundary.

\subsection{Avoidance trajectory by one arc}

If the obstacle is not on the vehicle's course as shown in Fig. 4, one left turn is enough to avoid both of the obstacle and road boundary. In this study, the following two trajectories are derived.

Trajectory $\mathrm{C}$ is an avoidance trajectory for the obstacle. It aims at restoring the yaw angle of the vehicle keeping the vehicle off the obstacle. Trajectory $\mathrm{C}$ is formulated with the following geometrical conditions.

(C-i) The arc $\mathrm{O}_{\mathrm{V}} \mathrm{F}$ is circumscribed to the vehicle direction line $\mathrm{L}_{\mathrm{V}}$.

(C-ii) The arc $\mathrm{O}_{\mathrm{V}} \mathrm{F}$ passes through the current vehicle position.

(C-iii) The off-limit circle $\mathrm{C}_{\mathrm{P}}$ is inscribed in the arc $\mathrm{O}_{\mathrm{V}} \mathrm{F}$.

From these conditions, the turning radius $R_{C}$ and the central angle of the arc $\theta_{C}$ are solved as: 


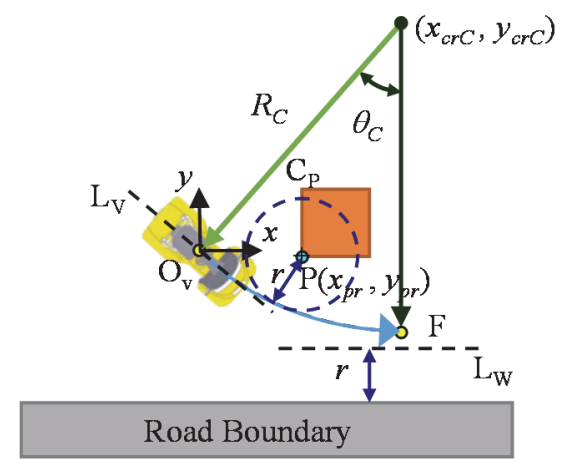

(a) Trajectory C

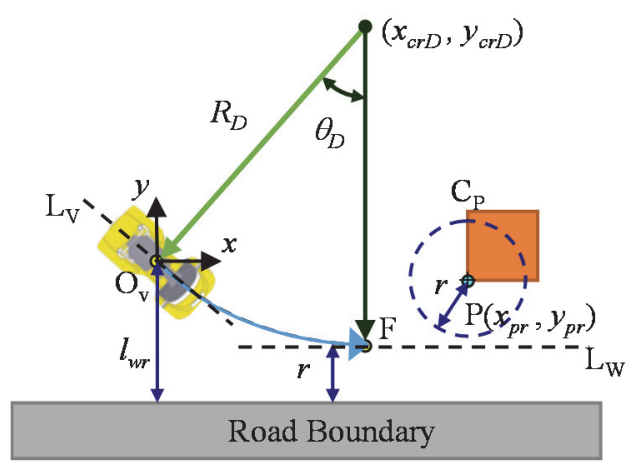

(b) Trajectory D

Fig. 4 Geometric description of the avoidance trajectory by one arc.

$$
R_{C}=\frac{x_{p r}^{2}+y_{p r}^{2}-r^{2}}{2\left(r-x_{p r} \sin \theta_{v}-y_{p r} \cos \theta_{v}\right)}, \quad \theta_{C}=\theta_{v} .
$$

Trajectory $\mathrm{C}$ is available when the finishing point is more than $r$ away from the road boundary. This is expressed as:

$$
R_{C}\left(1-\cos \theta_{v}\right) \leq l_{w r}-r
$$

Trajectory D is an avoidance trajectory for the road boundary. It aims at restoring the yaw angle of the vehicle keeping the vehicle off the road boundary. Trajectory $\mathrm{D}$ is formulated with the following geometrical conditions.

(D-i) The arc $\mathrm{O}_{\mathrm{v}} \mathrm{F}$ is circumscribed to the vehicle direction line $\mathrm{L}_{\mathrm{V}}$,

(D-ii) The arc $\mathrm{O}_{\mathrm{v}} \mathrm{F}$ passes through the current vehicle position.

(D-iii) The arc $\mathrm{O}_{\mathrm{vF}}$ is circumscribed to the off-limit line $\mathrm{L}_{\mathrm{w}}$.

The radius $R_{D}$ and the central angle $\theta_{D}$ are derived from these conditions as follows:

$$
R_{D}=\frac{l_{w r}-r}{1-\cos \theta_{v}}, \quad \theta_{D}=\theta_{v}
$$

Trajectory $\mathrm{D}$ is available unless the off-limit circle $\mathrm{C}_{\mathrm{P}}$ intersects the off-limit line $\mathrm{L}_{\mathrm{w}}$. This condition is expressed as:

$$
y_{p r}-r>-l_{w r} .
$$

\section{Redesign of the autonomous frontal obstacle avoidance system}

\subsection{Outline of the proposed system}

In this chapter, the autonomous frontal obstacle avoidance system is redesigned to build avoidance trajectory updating function into it. Figure 5(a) shows the flow chart of the proposed system. This system consists of four subsystems: surroundings recognition, obstacle position prediction, avoidance trajectory generation and desired steering angle profile generation. The following subsections describes them in detail. The system is supposed to be activated if an obstacle is on the vehicle's course and the time-to-collision is less than 2.0 seconds. Although the system should include a decision branch to execute braking without steering like automatic emergency braking, this study does not implement it since the main theme of this study is collision avoidance by steering.

Figure 5(b) shows the flow chart of the previous system proposed by the authors (Hayashi et al., 2012), as a reference. Avoidance by braking is omitted in the figure. The difference is that the previous system calculates avoidance trajectory only once at the start of avoidance, that is, it does not update the avoidance trajectory after starting autonomous avoidance.

\subsection{Surrounding recognition}

It has been reported in the authors' previous study (Hayashi et al., 2012) that the obstacle and road boundaries can 


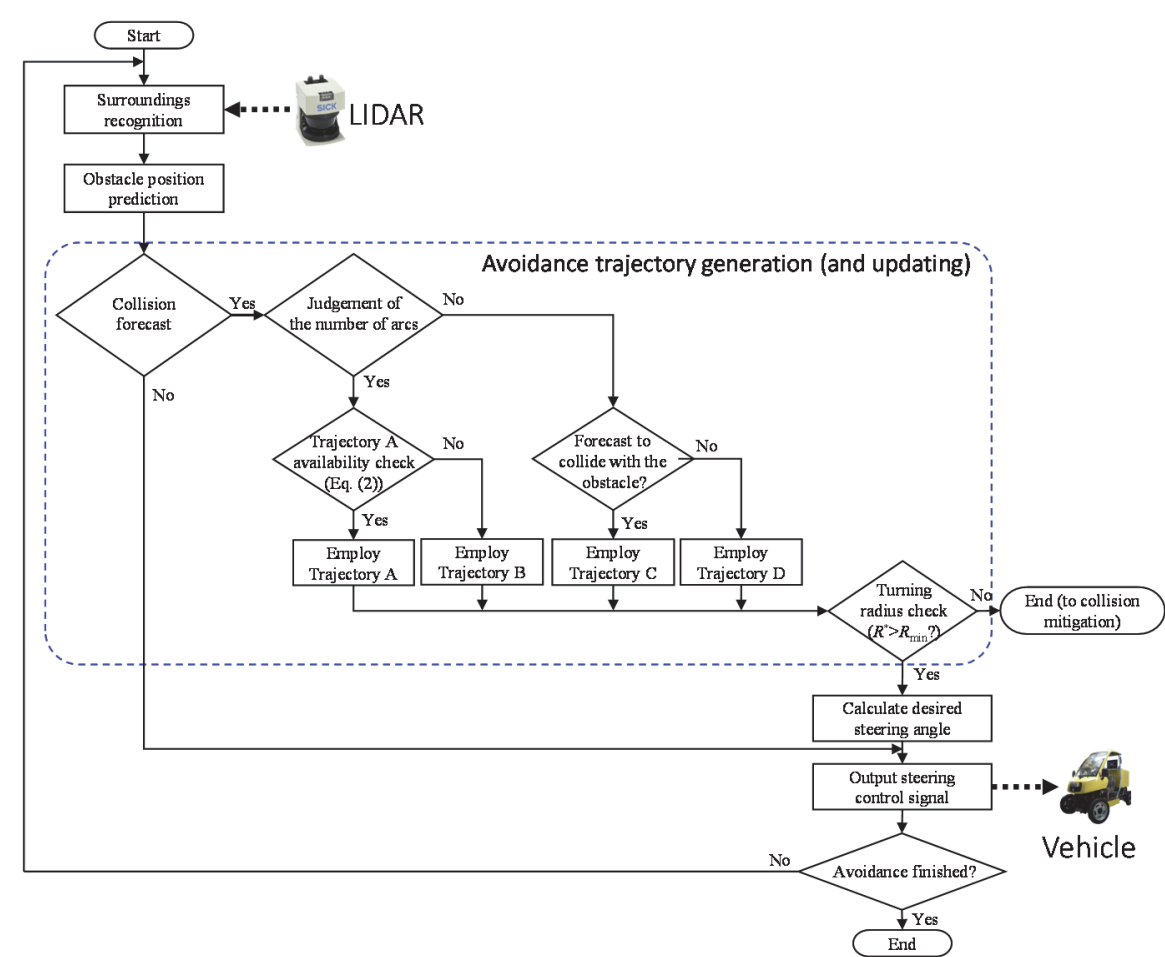

(a) the proposed system

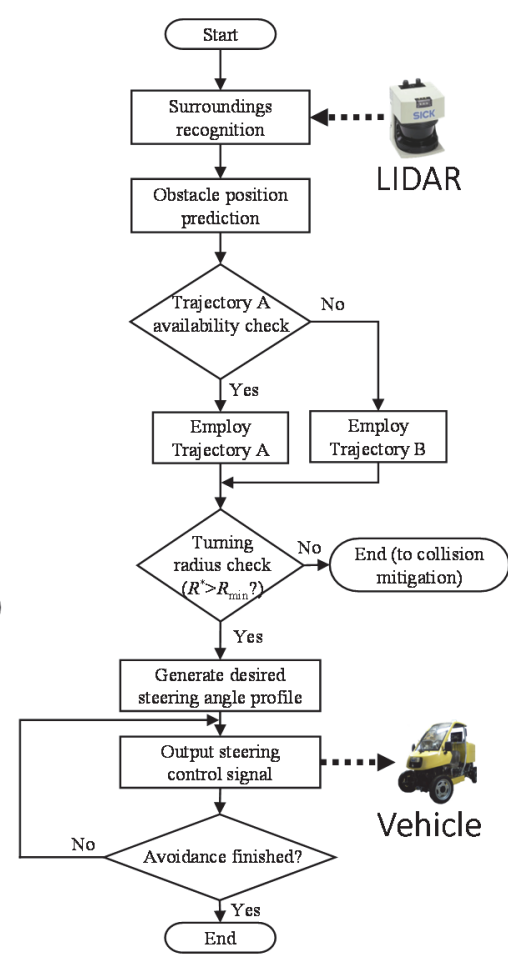

(b) the previous system

Fig. 5 Flow chart of the frontal obstacle avoidance system. The proposed system has a trajectory updating function, whereas the previous system generates avoidance trajectory only once.

be detected and the position of them can be measured by using LIDAR if they are in front of the vehicle, though the method is a simple one. The system may use a more advanced techniques to detect an obstacle and measure the position and speed of it, in order to improve the accuracy of collision avoidance. In this paper, since the main purpose of this study is to verify the updating method of the avoidance trajectory, surroundings recognition subsystem is omitted and the position of the obstacle $\left(X_{o b}, Y_{o b}\right)$ and the road boundary $L_{w r}$ (see Fig. 1) are directly given to the obstacle position prediction subsystem. In addition, the speed of the obstacle $\left(V_{o b x}, V_{o b y}\right)$ is calculated from the difference of position of the obstacle in 0.2 seconds.

\subsection{Obstacle position prediction}

To deal with moving obstacles like pedestrians, the authors' previous studies (Hayashi et al., 2012) have proposed to utilize predicted position of the obstacle to generate the avoidance trajectory instead of the current position of the obstacle. The prediction procedure is as follows.

First, the time to collision $T_{t o}$ is calculated under assumption that the obstacle moves at the constant speed and the vehicle moves straightforward decelerating with a constant deceleration. $T_{t o}$ is expressed as:

$$
T_{t o}(t)=\frac{-V_{v}(t) \cos \theta_{t}(t)+V_{o b x}(t)+\sqrt{\left(V_{v}(t) \cos \theta_{t}(t)-V_{o b x}(t)\right)^{2}+2 a_{v x}(t) \cos \theta_{t}(t)\left(X_{o b}(t)-X_{v}-l_{h}\right)}}{a_{v x}(t) \cos \theta_{t}(t)},
$$

where $X_{v}$ is the position of the vehicle, $V_{v}$ is the vehicle speed, $a_{v x}$ is acceleration of the vehicle and $l_{h}$ is the distance between the center of gravity of the vehicle and the front end of the vehicle. Then, the obstacle position at the time instant when the vehicle collide with the obstacle is predicted by the following equation:

$$
X_{p r}(t)=X_{o b}(t)+V_{o b x}(t) T_{t o}(t), Y_{p r}(t)=Y_{o b}(t)+V_{o b y}(t) T_{t o}(t) .
$$

Avoidance trajectories are generated against the predicted obstacle position $\left(X_{p r}, Y_{p r}\right)$ expressed above.

Since this is also a simple and basic prediction method using first order prediction, the predicted position of the obstacle is not necessarily accurate. However, the inaccuracy is compensated by the trajectory updating method described below. Alternatively, the system may use a more advanced techniques to predict an obstacle's motion. 


\subsection{Avoidance trajectory generation (and updating)}

This subsystem updates the avoidance trajectory using the theory proposed in Chapter 3 to accommodate change of behavior of the obstacle. The avoidance trajectory is to be revised only when necessary. Therefore, whether the current avoidance trajectory causes collision or not has to be forecast before revising the avoidance trajectory.

\subsubsection{Collision forecast}

A collision is forecast to occur if the predicted obstacle position or the road boundary is on the vehicle's course. Since it is assumed to be impossible to avoid the obstacle by evading leftward, the vehicle is forecast to collide with the obstacle if the right edge of the obstacle $\left(X_{p r}, Y_{p r}\right)$ is in the shaded area in Fig. 6(a). Though $r$ has been defined as the half of the vehicle width in Chapter 3, to add an extra margin of safety, $r$ is set at $0.5475 \mathrm{~m}$, including a margin of $0.05 \mathrm{~m}$.

In addition, the vehicle is forecast to collide with the road boundary if the finishing point of the avoidance trajectory $F$ is in the shaded area. This can occur because the vehicle cannot follow the avoidance trajectory strictly due to the dynamics of the vehicle and its steering system.

If the current avoidance trajectory is forecast to cause a collision with the obstacle or the road boundary, the system update the avoidance trajectory by the following method. Otherwise, the system continues to use the current trajectory. Then, the current turning radius $R^{*}$ and central angle $\theta^{*}$ is not updated.

\subsubsection{Judgement of the number of arcs}

If revision of avoidance trajectory is necessary, the system determines the number of arcs needed, utilizing Trajectory D. Since trajectory D is the largest arc which does not cause collision with the road boundary, if trajectory D cause a collision with the obstacle, it means that one arc is not enough to avoid collision. Hence, the avoidance trajectory must be composed of two arcs then. Figure 7(a) depicts the case which requires two arcs. Otherwise, one arc is enough to avoid both of the obstacle and road boundary as shown in Fig. 7(b).

\subsubsection{Availability check of trajectory A}

If the number of arcs necessary for collision avoidance is two, the system check if trajectory $\mathrm{A}$ is available or not using Eq. (2). If trajectory $\mathrm{A}$ is available, the radius and central angle of avoidance trajectory arc are updated as $R^{*}=R_{A}$ and $\theta^{*}=\theta_{A}$. Otherwise, the system employs trajectory $\mathrm{B}$, updating them as $R^{*}=R_{B}$ and $\theta^{*}=\theta_{B}$.

\subsubsection{Availability check of trajectory $C$ (branching by forecast collision object)}

If the number of arcs is one, the system checks whether the forecast collision object is the obstacle or the road boundary. If the forecast collision object is the obstacle, it means that the radius of the avoidance trajectory is too small. Therefore, the radius of the avoidance trajectory arc should be increased. In this study, to minimize the amount of revision, trajectory $\mathrm{C}$ is employed and the radius and central angle of avoidance trajectory arc are updated as $R^{*}=R_{C}$ and $\theta^{*}=\theta_{C}$. On the contrary, if the forecast collision object is the road boundary, it means that the radius of the avoidance trajectory is too large. Therefore, trajectory $\mathrm{D}$ is employed to decrease the radius of the avoidance trajectory arc and the radius and central angle of avoidance trajectory arc are updated as $R^{*}=R_{D}$ and $\theta^{*}=\theta_{D}$.

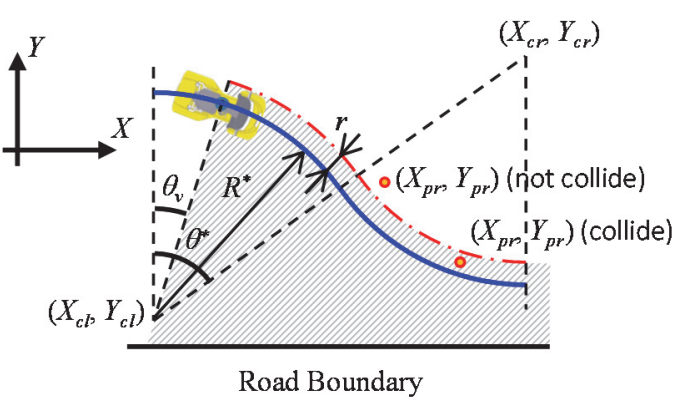

(a) Collision with the obstacle

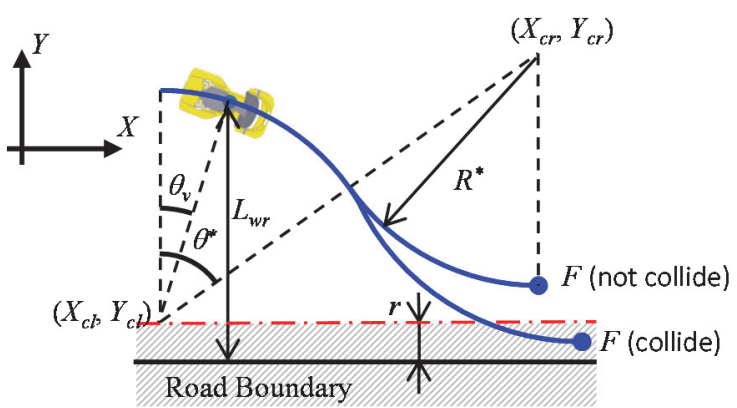

(b) Collision with the road boundary

Fig. 6 Forecast of collision. If the obstacle in the shaded area in (a), the vehicle is forecast to collide with the obstacle. If the finishing point is in the shaded area in (b), the vehicle is forecast to collide with the road boundary. 


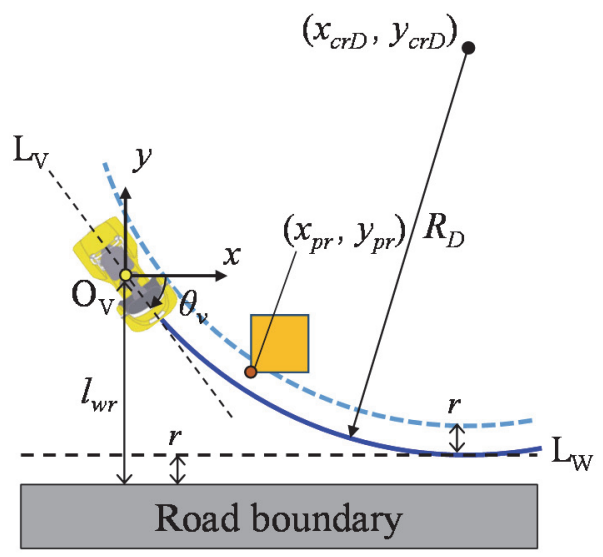

(a) Need two arcs

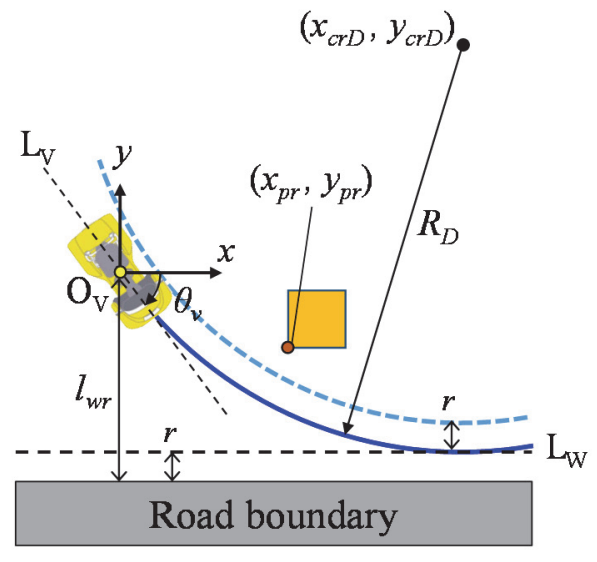

(b) Need one arc

Fig. 7 The number of arcs to evade an obstacle. If the edge of the obstacle is inside of the blue broken line, one arc is enough to avoid both of the obstacle and the road boundary. Otherwise, two arcs are needed.

\subsubsection{Turning radius check}

After the avoidance trajectory is revised, the system checks if the radius of the trajectory arc is larger than the minimum turning radius $R_{\min }$. If the radius is smaller than $R_{\min }$, the vehicle cannot follow the avoidance trajectory even with its maximum steering angle. It means that a collision with the obstacle or the road boundary is physically inevitable. In this case, the vehicle should take an appropriate collision mitigation measure. Since it is not the main theme of this study to discuss it, collision mitigation function is omitted and the system continues to decelerate the vehicle holding current steering angle.

\subsection{Generation of desired steering angle}

the desired steering angle $\delta_{s w}{ }^{*}$ is recalculated using the updated radius $R^{*}$ as follows:

$$
\delta_{s w}^{*}=\sigma \frac{1+K V_{0}^{2}}{N} \frac{l}{R^{*}},
$$

where $V_{0}$ is initial vehicle speed, which is supposed to be obtained by wheel speed sensors equipped in the vehicle. $K, N$, $l$ are the stability factor, steering gear ratio and wheelbase of the vehicle, respectively. Their values have been shown in Table $1 . \sigma=-1$ if the employed trajectory is trajectory A or B and it is replaced with 1 to switch turning direction from right to left after $\theta_{v}$ reaches $\theta^{*}$. If the employed trajectory is $\mathrm{C}$ or $\mathrm{D}, \sigma=1$. For all trajectories, once $\theta_{v}$ reaches zero, $\sigma$ is kept at $\sigma=0$. In addition to steering, the vehicle is decelerated with maximum deceleration $\left(-2 \mathrm{~m} / \mathrm{s}^{2}\right)$ to stop promptly since the supposed scenes are emergency avoidance scenes.

\section{Verification by numerical simulation}

\subsection{Dynamics model of the vehicle}

\subsubsection{Vehicle model}

Numerical simulation are executed to verify the validity and effectiveness of the redesigned system. The bicycle model shown in Fig. 8 is used as the vehicle model. $x$ and $y$ are the moving coordinate whose origin is the center of gravity of the vehicle. $\Psi$ is the yaw angle of the vehicle. The equation of motion is expressed as:

$$
m(\dot{u}-v \gamma)=-F_{s f} \sin \delta_{f}+F_{d r}, \quad m(\dot{v}+u \gamma)=F_{s f} \cos \delta_{f}+F_{s r}, \quad I_{z} \dot{\gamma}=l_{f} F_{s f} \cos \delta_{f}-l_{r} F_{s r},
$$

where $u$ and $v$ are longitudinal and lateral velocity of the vehicle. $\gamma$ is yawrate, which equals to $\dot{\Psi}$. $\delta_{f}$ is steering angle of the front tire, $F_{s f}$ and $F_{s r}$ are the side forces of front and rear tire and $F_{d r}$ is the driving force of the rear tires. $F_{s f}, F_{s r}$ and $F_{d r}$ are expressed as: 


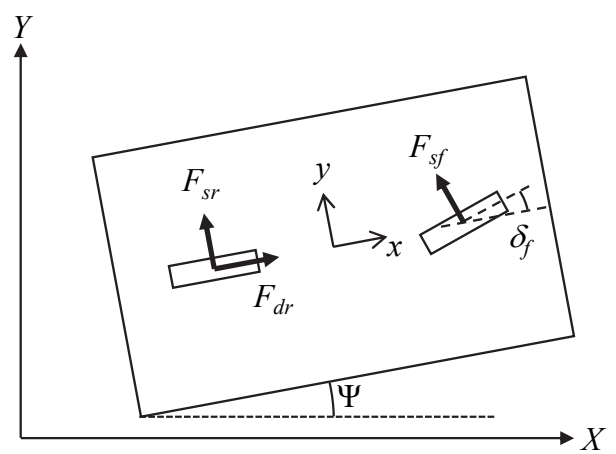

Fig. 8 Vehicle model (bicycle model).

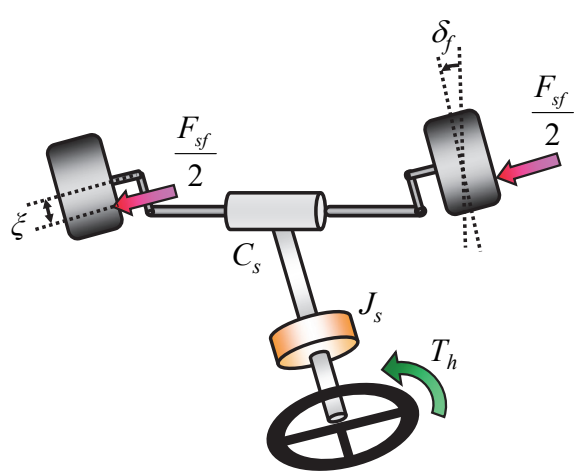

Fig. 9 Steering system model.

$$
F_{s f}=2 C_{f}\left(\delta_{f}-\tan ^{-1} \frac{v+l_{f} \gamma}{u}\right), \quad F_{s r}=2 C_{r}\left(-\tan ^{-1} \frac{v-l_{r} \gamma}{u}\right), \quad F_{d r}=m a_{v x}
$$

where $C_{f}$ and $C_{r}$ are cornering stiffness of front and rear tires.

\subsubsection{Steering system model}

The steering system is simulated by the dynamics model shown in Fig. 9. The equation of motion in terms of steering wheel angle $\delta_{s w}$ is expressed as:

$$
J_{s} \ddot{\delta}_{s w}=-C_{s} \dot{\delta}_{s w}+2 \xi C_{f}\left(\delta_{s w}-N \tan ^{-1} \frac{v+l_{f} \gamma}{u}\right)+N^{2} T_{h}
$$

where $J_{s}$ is moment of inertia of the steering system, $C_{s}$ is viscous damping coefficient of the steering system and $\xi$ is pneumatic trail. Their values are $J_{s}=13 \mathrm{Nm}^{2}, C_{s}=140 \mathrm{Ns} / \mathrm{rad}$ and $\xi=0.06 \mathrm{~m}$. $N$ is steering gear ratio, whose value is shown in Table $1 . T_{h}$ is steering torque applied by a servo motor. The control law of the steering angle is as follows:

$$
T_{h}= \begin{cases}4.9 \mathrm{~N} \cdot \mathrm{m} & \left(\delta_{s w}^{*}-\delta_{s w} \geq 2 \mathrm{deg}\right), \\ 0 \mathrm{~N} \cdot \mathrm{m} & \left(2 \mathrm{deg}>\delta_{s w}^{*}-\delta_{s w} \geq-2 \mathrm{deg}\right), \\ -4.9 \mathrm{~N} \cdot \mathrm{m} & \left(-2 \mathrm{deg}>\delta_{s w}^{*}-\delta_{s w}\right) .\end{cases}
$$

The control method of the actual steering servo controller is closed to the public. Therefore, it may be different from this kind of controller. However, it has been reported that the response of the steering system in the simulations with the bangbang control law agrees well with that of the actual experimental vehicle (Hayashi et al., 2011).

\subsection{Vehicle trajectory and time history data of the simulation results}

\subsubsection{Simulation conditions}

In this section, numerical simulations are executed under two conditions about the obstacle. The time history position of the obstacle in each conditions are shown in Fig. 10 and 11. Figure 10 indicates the obstacle cross the road from north to south with a constant speed. Figure 11 indicates that the obstacle moves southward at first and veers southwestward after 1.0 seconds. Road width is $4 \mathrm{~m}$ and $5 \mathrm{~m}$, respectively. The initial speed of the vehicle is $8 \mathrm{~m} / \mathrm{s}$ and autonomous avoidance system is activated at 0 second in both conditions.

\subsubsection{Simulation results}

Figures 12 and 13 show the simulation result for condition 1. Fig. 12 is the result of the previous system and Fig. 13 is the result of the proposed system. In each figure, (a) shows the trajectories of the vehicle and the obstacle. Yellow squares and cyan (magenta) rectangles show the position and posture of the obstacle and the vehicle of every 0.2 second, respectively. However, the obstacles have not been drawn after the vehicle passed the obstacle. Therefore, if a yellow square has an overlap with a cyan (magenta) rectangle, it indicates that the vehicle collides with the obstacle. Each figure 
(b) shows the time history data of avoidance trajectory, desired turning radius and steering angle. In the graphs of avoidance trajectory, A to D mean that the avoidance trajectory is revised by each of the avoidance trajectory and $\mathrm{H}$ means that the avoidance trajectory is not revised but the current trajectory is inherited.

Figure 12(a) indicates that the previous system causes a collision with the obstacle. It is mainly due to the response delay of the steering system as shown in the bottom graph of Fig. 12(b). Inaccuracy of obstacle position prediction expressed by Eq. (12) is also a cause of the collision. The obstacle position is predicted by assuming that the vehicle moves straight, whereas the actual vehicle turns right to evade the obstacle. Therefore, $T_{t o}$ calculated by Eq. (11) is not accurate. However, it is impossible to predict an accurate obstacle position since the motion of the ego-vehicle is determined based on the predicted obstacle position. Therefore, the assumption must be permissible.

On the other hand, Fig. 13(a) indicates that the proposed system avoids the obstacle without collision. The top graph of Fig. 13(b) indicates that the system continues revising avoidance trajectory until it passes the obstacle. The middle graph indicates that turning radius is decreased to compensate the delay of the steering system and inaccuracy of the prediction. These results indicate that the proposed system is more effective even for obstacles with constant motion.

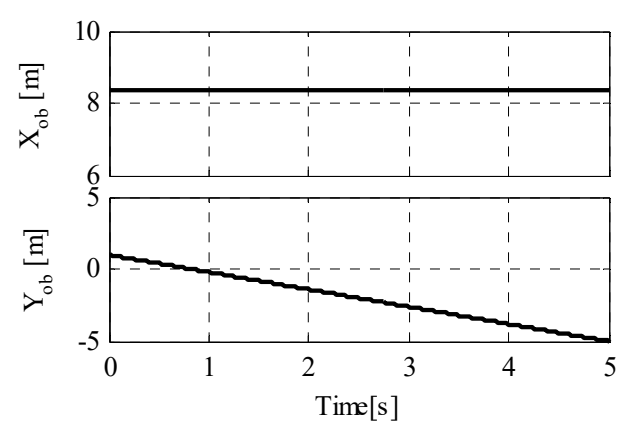

Fig. 10 Obstacle position (Condition 1). It moves at a constant speed.

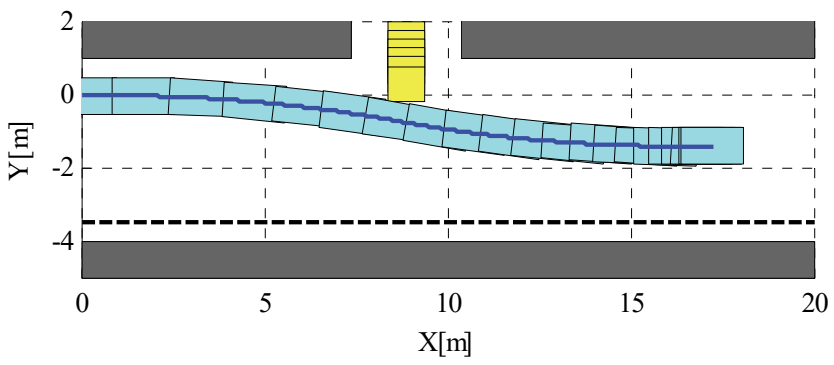

(a) Trajectories of the vehicle and obstacle

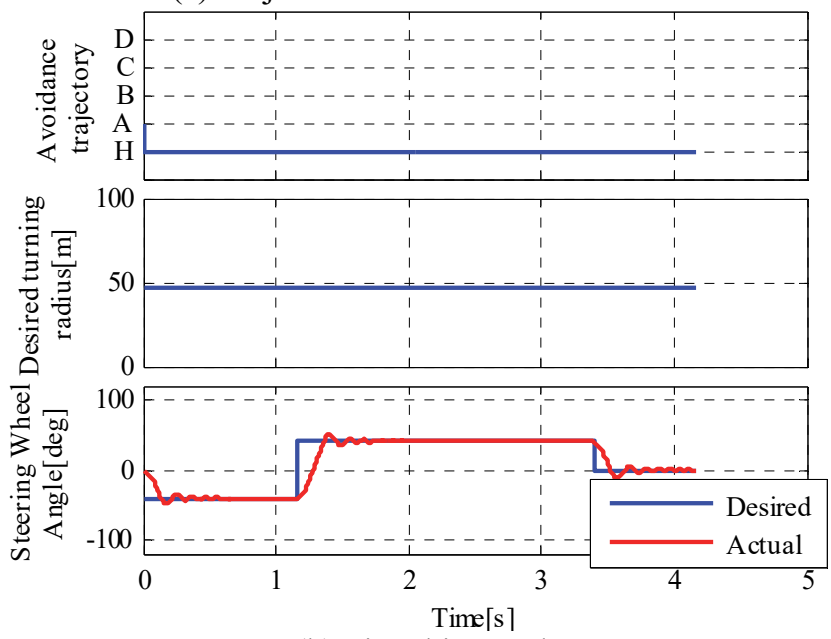

(b) Time history data

Fig. 12 Simulation result (the previous system, condition 1). The vehicle collides with the obstacle because of the delay of the steering system.

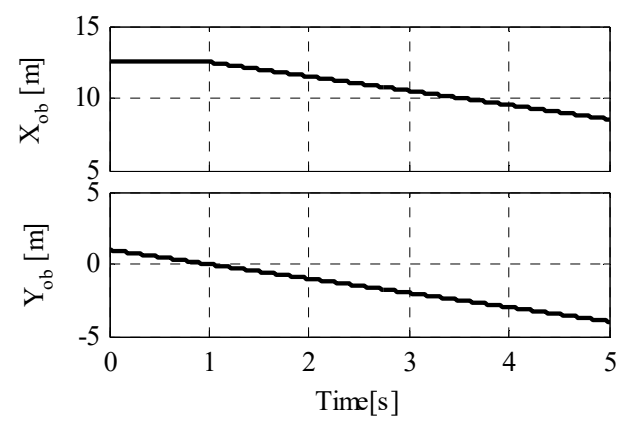

Fig. 11 Obstacle position (Condition 1). The obstacle's motion changes at 1.0 second.

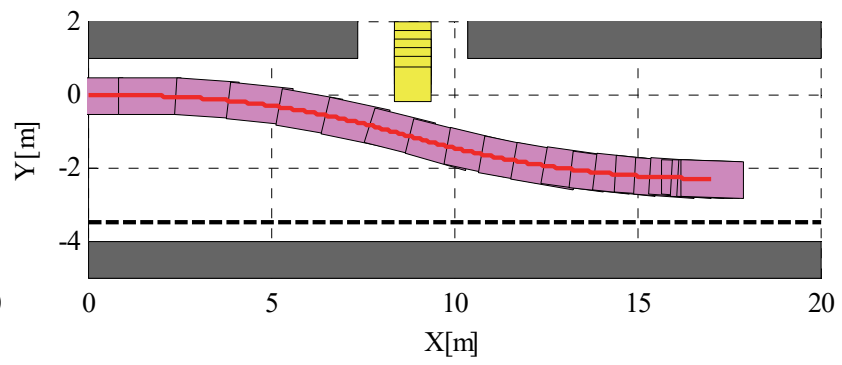

(a) Trajectories of the vehicle and obstacle
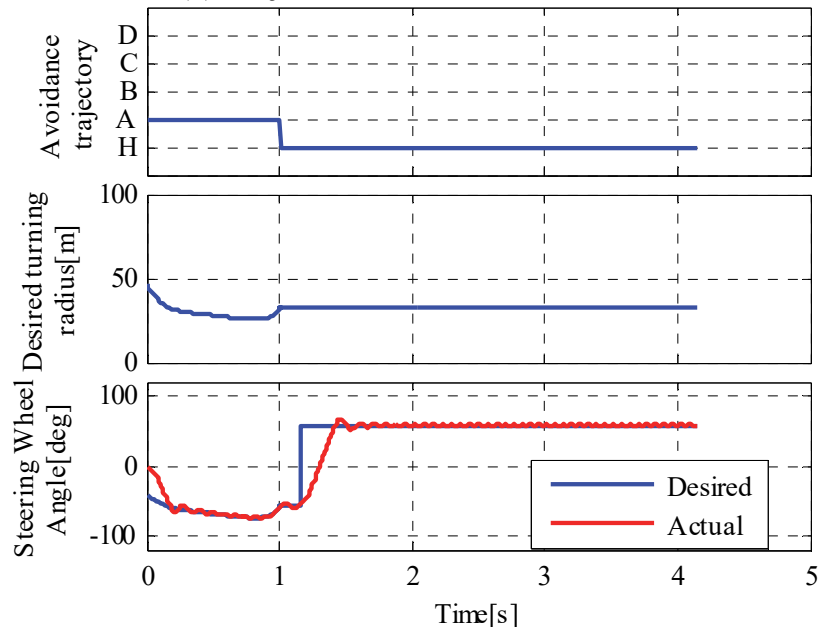

(b) Time history data

Fig. 13 Simulation result (the proposed system, condition 1). The proposed system successfully avoids the obstacle without having a collision with the road boundary. 


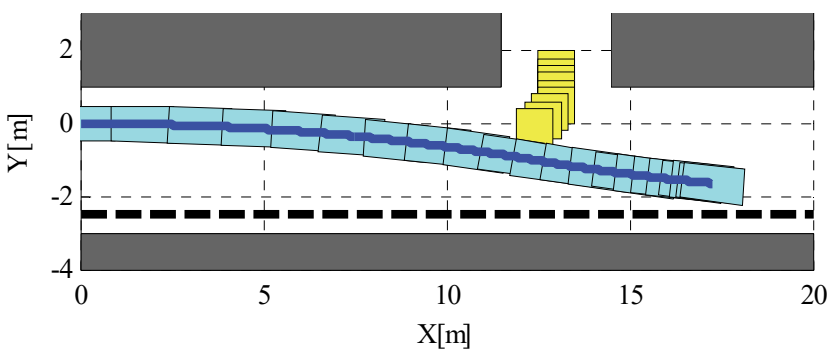

(a) Trajectories of the vehicle and obstacle

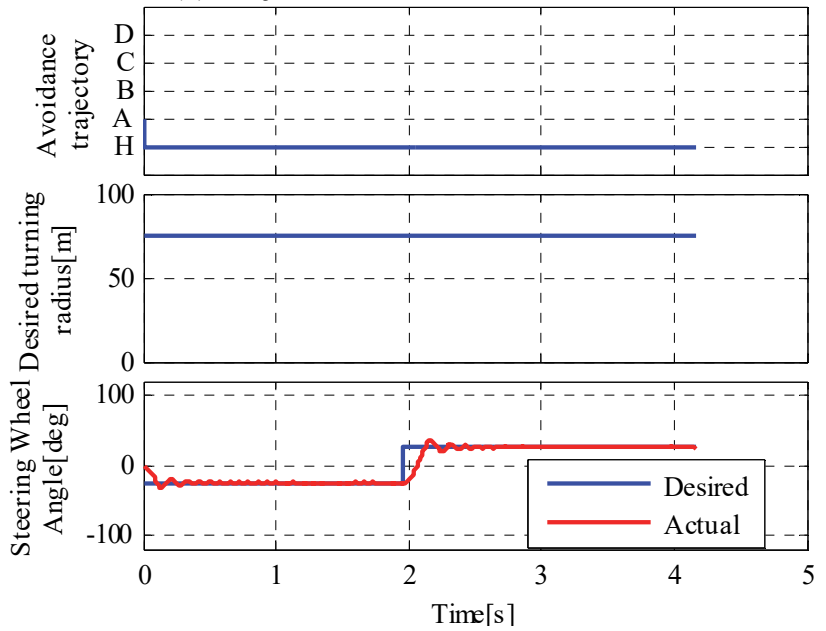

(b) Time history data

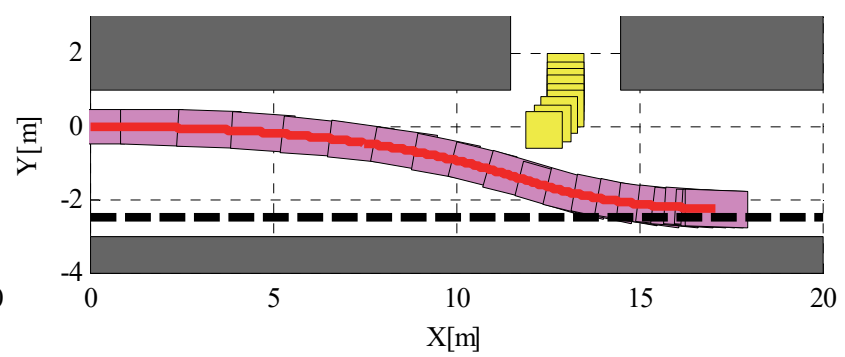

(a) Trajectories of the vehicle and obstacle
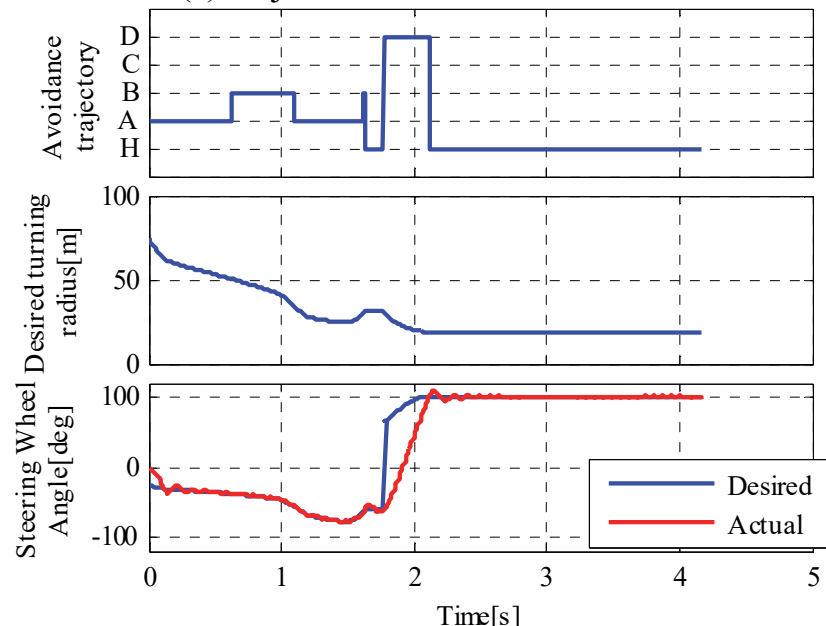

(b) Time history data

Fig. 14 Simulation result (the previous system, condition Fig. 15 Simulation result (the proposed system, condition 2). The vehicle collides with the obstacle because of the obstacle's motion changes during avoidance.

2). The proposed system successfully avoids the obstacle by updating the avoidance trajectory.

Figures 14 and 15 show the results for condition 2. In this condition, change of the obstacle motion also give rise to necessity of revising avoidance trajectory. Figure 14(a) indicates that the previous system causes a collision with the obstacle also in this condition. Condition of collision is even worse. On the other hand, Fig. 15(a) indicates that the proposed system succeeds in collision avoidance with the obstacle and the road boundary. The top graph of Fig. 15(b) indicates that the system revises avoidance trajectory using avoidance trajectory B before the change of motion of the obstacle at 1.0 second since the road is narrower in this condition. After evading the obstacle, the system revises avoidance trajectory by using avoidance trajectory $\mathrm{D}$ in order to avoid collision with the road boundary. The middle graph of the Fig. 15(b) indicates that the system decreases the turning radius rapidly to respond to the change of motion of the obstacle at 1.0 second. These results indicate that the proposed system is valid and effective to the obstacles which changes its motion during avoidance.

\subsection{Basic overall evaluation of performance improvement by Monte Carlo method}

In this section, an overall evaluation is carried out in order to assess the performance improvement by the proposed system quantitatively. Time history simulations are executed 1,000 times varying obstacle motion and road width at random within the range shown in Table 2. The simulations does not include the conditions that the obstacle blocks the space for the ego-vehicle to pass through due to the assumed condition of target scenes (4).

In this chapter, the half of the vehicle width with safety margin $r$ for the previous system is set at $0.8 \mathrm{~m}$, which is the value used in the previous study (Hayashi et al., 2012) to compensate the delay of the steering system.

Figure 16 shows the result of the assessment. As can be seen in Fig. 16, the proposed system increases the success rate from $72.0 \%$ to $91.4 \%$ despite less safety margin. Moreover, the proposed system collides mainly with road boundaries even in cases of failure, whereas the previous system collides only with the obstacle such as pedestrians. Therefore, in terms of avoidance of obstacles, the success rate of the proposed system is $99.0 \%$. Though it is arguable which is better to collide if the collision is inevitable, it is sure that the proposed system improves the collision avoidance performance, leading to the conclusion that the proposed trajectory updating method is valid and effective. 
Table 2 Range of parameters about obstacle's motion.

\begin{tabular}{|c|c|c|l|l|}
\hline Parameters & Min. & Max. & \multicolumn{1}{|c|}{ Unit } & \multicolumn{1}{|c|}{ Remarks } \\
\hline Appearance position (Initial $X_{o b}$ ) & 6 & 10 & $\mathrm{~m}$ & equivalent to $0.75-1.25 \mathrm{sec}$ as initial TTC \\
\hline Speed before change of motion & 1.0 & 2.0 & $\mathrm{~m} / \mathrm{s}$ & \\
\hline Angle before change of motion & -45 & 45 & $\mathrm{deg}$ & 0deg indicates negative Y direction. \\
\hline Time of change of motion & 0.75 & 1.25 & $\mathrm{sec}$ & \\
\hline Speed after change of motion & 0.0 & 2.0 & $\mathrm{~m} / \mathrm{s}$ & \\
\hline Angle after change of motion & -45 & 45 & $\mathrm{deg}$ & 0deg indicates negative Y direction. \\
\hline Road width & 4.0 & 8.0 & $\mathrm{~m}$ & \\
\hline
\end{tabular}

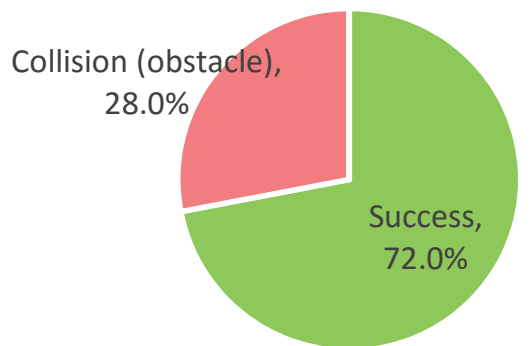

(a) The previous system $(r=0.8 \mathrm{~m})$

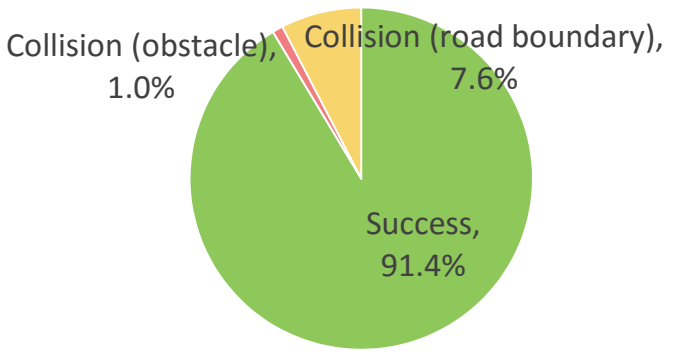

b) The proposed system $(r=0.5475 \mathrm{~m})$

Fig. 16 Success rate of collision avoidance. The collision avoidance performance of the proposed system is higher than that of the previous system.

\section{Conclusion}

This paper proposed an autonomous frontal avoidance system with trajectory updating function, by building a regeneration method of avoidance trajectory which starts from the vehicle position in the middle of avoidance into the previously proposed system. The redesigned system is examined by numerical simulations to evaluate the validity and effectiveness. The result indicates that the proposed trajectory updating method is valid and effective.

The future work is to implement the propose system in the vehicle shown in Fig. 2 and carry out demonstration experiments using the real car.

\section{References}

Fujioka, T., Shibata, A., Tsukasaki, Y. and Sawada, S., Vehicle motion control for minimizing collision risk by use of optimal control theory, (2008 JSAE Spring Convention) Proceedings(In Japanese), No.8-08 (2008), pp.21-26.

Glaser, S., Vanholme, B. and Mammar, S., Maneuver-Based Trajectory Planning for Highly Autonomous Vehicles on Real Road With Traffic and Driver Interaction, IEEE Transactions on Intelligent Transportation Systems, Vol. 11, No. 3 (2010), pp. 589-604.

Hayashi, R., Isogai, J., Fujita, S., Raksincharoensak, P. and Nagai M., Development of autonomous forward obstacle avoidance system by using in-wheel-motor and steering control of micro electric vehicle, JSAE Transactions (In Japanese), Vol. 42, No. 1 (2011), pp. 87-93.

Hayashi, R., Isogai, J., Raksincharoensak, P. and Nagai, M., Autonomous collision avoidance system by combined control of steering and braking using geometrically optimized vehicular trajectory, Vehicle System Dynamics: International Journal of Vehicle Mechanics and Mobility, No. 50, Supp. 1 (2012), pp.151-168, DOI: 10.1080/00423114.2012.672748.

Horiuchi, S., Hirao, R., Okada, K., and Nohtomi, S., Optimal steering and braking control in emergency obstacle avoidance, Transactions of the Japan Society of Mechanical Engineers, Series C (In Japanese), Vo. 72, No. 722 (2006), pp. 180-185.

Institute of Traffic Accident Research and Data Analysis, Analysis of human factors in crossing collisions, ITARDA information, No. 56 (2005). Available at http://www.itarda.or.jp/itardainfomation/english/info56/56top.html.

Kimura, K., Nonaka, K. and Sekiguchi, K., Real-time model predictive obstacle avoidance control for vehicles with 
reduced computational effort using constraints of prohibited region, Mechanical Engineering Journal, Vol. 2 , No. 3 (2015), 14-00568, DOI:10.1299/mej.14-00568

Llorca, D. F., Milanes, V., Alonso, I. P., Gavilan, M., Daza, I. G., Perez, J. and Sotelo, M. A., Autonomous Pedestrian Collision Avoidance Using a Fuzzy Steering Controller, IEEE Transactions on Intelligent Transportation Systems, Vol. 12, No. 2 (2011), pp. 390-401.

Ohmuro, A. and Hattori, Y., Optimum vehicle trajectory control for obstacle avoidance - a minimax problem of resultant vehicle force, Transactions of the Japan Society of Mechanical Engineers, Series C (In Japanese), Vol. 76, No. 772 (2010), pp. 3587-3594.

Shibata, N., Sugiyama, S. and Wada, T., Collision avoidance control with steering using velocity potential field, Proceedings of IEEE Intelligent Vehicles Symposium Proceedings (2014), pp.438-443.

Shimizu, M., Usami, M. and Fujinami, H., Development of Collision-Avoidance Assist System (2007 JSAE Autumn Convention) Proceedings (In Japanese), No. 148-07 (2007), pp. 25-30.

Soudbakhsh, D., Eskandarian, A. and Chichka, D., Vehicle Collision Avoidance Maneuvers With Limited Lateral Acceleration Using Optimal Trajectory Control, Journal of Dynamic Systems, Measurement, and Control, Vol. 135 (2014), 041006-1. 